Polymer Journa1, Vol. 8, No. 3, pp 247-253 (1976)

\title{
Hypochromic Effects in UV Spectra of Polymers in Solution
}

\author{
Bernhard Stützel, Takeaki Mıуamoto, ${ }^{*}$ and Hans-Joachim Cantow \\ Institut für Makromolekulare Chemie der Universität Freiburg, \\ D-78 Freiburg, i. Br., Stefan-Meier Str. 31, West Germany. \\ (Received September 19, 1975)
}

\begin{abstract}
The hypochromic effect in the UV spectra of polystyrene and styrene copolymers in solution was investigated in detail. For this purpose, isotactic and syndiotactic-rich polystyrenes as well as their dimer model compounds, 2,4-diphenylpentanes, and styrene-methyl methacrylate copolymers were prepared. UV absorption measurements were carried out in dioxane and tetrahydrofuran at various temperatures. The extinction coefficients of phenyl rings depended not only on the solvent and the temperature but also on the microstructure of the polymers. A discontinuous temperature dependence of the extinction coefficients was observed between 40 and $50^{\circ} \mathrm{C}$ for homopolystyrenes as well as their dimer model compounds. It was suggested that this might be explained in terms of the rotation of phenyl rings. On the other hand, the hypochromicity in binary copolymers appeared to depend strongly on the sequential arrangement of monomers (the chain architecture and the chemical composition of copolymers). The measured average extinction coefficients were well interpreted by a simple treatment by using triad probability proposed by Harwood. The results were discussed in relation to the UV spectrometer which is being used as an auxiliary detector in gel-permeation chromatography and microcolumn chromatography.
\end{abstract}
KEY WORDS Hypochromic Effect / UV Spectrum / Extinction
Coefficient / 2,4-Diphenylpentane / Polystyrene / Styrene-Methyl
Methacrylate Copolymer / Sequential Arrangement /

The quantities of eluted homopolymers in gel-permeation chromatography (GPC) and microcolumn-solubility chromatography (MCC) can be determined through the detection of a single physical value, such as refractive index increment, which is proportional to the concentration of the polymer in the eluent. However, the determination of the chemical composition of a binary copolymer at a certain elution count (i.e., the point-by-point composition) requires another quantity to be specified. This can be performed by using an ultraviolet (UV) detector auxiliarily, so far as the extent of absorption at a certain wavelength is different for the two components of the copolymer. ${ }^{1,2}$

If the UV extinction coefficient, $\varepsilon$, depends solely on the population of the units able to absorb in the copolymer chains, the point-bypoint composition can be determined easily with such a dual detector system as mentioned above.

* Permanent address: Institute for Chemical Research, Kyoto University, Uji, Kyoto 611, Japan.
However, it has been observed for binary copolymers that the UV extinction coefficient depends not only on the population of the units able to absorb but also on the sequence length of copolymer chains. ${ }^{3,4}$ Moreover, it has been reported that temperature and steric isomerism also affect the extinction coefficient of UV absorption. ${ }^{5,6}$ In this report detailed studies are made on the various factors which may influence the UV absorption of phenyl rings; i.e., (1) the steric isomerism of polystyrene and its dimer model compound, (2) the temperature, (3) the chain architecture of styrene (ST) - methyl methacrylate (MMA) copolymers (i.e., alternating, random, and block with a fixed composition), and (4) the chemical composition of STMMA random copolymers.

\section{EXPERIMENTAL}

\section{Materials}

A stereoisomeric mixture of the 2,4-diphenylpentane was synthesized according to the method 
of Overberger, et al. ${ }^{7}$ Separation of meso- and $d, l$-isomer by preparative gas chromatography and characterization by NMR spectroscopy were based on the same procedures as used by Bovey, et al. ${ }^{8}$ The purity of $d, l$-isomer was $97 \%$ and that of meso-isomer was better than $88 \%$.

Isotactic polystyrene was polymerized with $\mathrm{Al}\left(\mathrm{C}_{2} \mathrm{H}_{5}\right)_{3}-\mathrm{TiCl}_{4}$ in heptane at $70^{\circ} \mathrm{C}$. The product was extracted with methyl ethyl ketone ${ }^{9}$ and the insoluble fraction used as isotactic sample (I-PS).

The I-PS sample was found to be highly isotactic through comparison of its IR and NMR spectra with those reported for isotactic polymers. ${ }^{8,9}$ The syndiotactic-rich sample (S-PS) was prepared by a radical polymerization technique. The molecular weights of these samples estimated from the maximum peak position in GPC were $20 \times 10^{4}$ and $16 \times 10^{4}$, respectively.

The alternating ST-MMA copolymer (SM$\mathrm{AL})$ was polymerized at $-78^{\circ} \mathrm{C}$ with $\mathrm{Al}\left(\mathrm{C}_{2} \mathrm{H}_{5}\right)_{1.5}$. $\mathrm{Cl}_{1.5}$ in toluene according to the method given by Hirooka, et al. ${ }^{11}$ The block copolymer (SMBlock) was kindly provided by Dr. T. FukudaTanaka, University of Kyoto, the preparation and characterization of which are given in ref 10. Random ST-MMA copolymer samples with different ST contents (coded as SM-series) were supplied by the courtesy of Dr. R.J. Brüssau, BASF AG., Ludwigshafen. These

Table I. Characterization data of ST-MMA copolymer samples used

\begin{tabular}{lccccccc}
\hline & \multirow{2}{*}{$\begin{array}{c}\text { ST, } \\
\text { Sample }\end{array}$} & $\begin{array}{c}\text { ST } \\
\text { mol } \%\end{array}$ & $\begin{array}{c}\text { Run } \\
\text { num- }\end{array}$ & & \multicolumn{2}{c}{ Triad probability } \\
\cline { 6 - 8 } & & & ber & & $f_{\text {SSS }}$ & $f_{\text {MSS }}$ & $f_{\text {MSM }}$ \\
\hline SM-20 & 22.3 & 14.0 & 43.38 & 0.0007 & 0.0533 & 0.9458 \\
SM-30 & 33.1 & 14.0 & 56.40 & 0.0220 & 0.2531 & 0.7252 \\
SM-40 & 41.0 & 14.0 & 61.86 & 0.0607 & 0.3712 & 0.5679 \\
SM-50 & 50.0 & 15.0 & 63.42 & 0.1339 & 0.4640 & 0.4021 \\
SM-60 & 59.0 & 15.0 & 60.37 & 0.2388 & 0.4998 & 0.2614 \\
SM-70 & 69.2 & 16.0 & 50.82 & 0.4004 & 0.4647 & 0.1349 \\
SM-80 & 79.4 & 16.0 & 37.03 & 0.5879 & 0.3576 & 0.0545 \\
SM-90 & 90.8 & 16.0 & 17.92 & 0.8183 & 0.1779 & 0.0097 \\
SM-AL & 50.0 & 40.0 & 100 & 0 & 0 & 1 \\
SM- & 48.1 & $13.5^{b}$ & 0 & 1 & 0 & 0 \\
Block & & & & & &
\end{tabular}

a Estimated from the maximum peak position in GPC by using a calibration curve for polystyrenes.

b Number-average molecular weight by osmometry. samples were low conversion products prepared by a radical copolymerization method. All the samples were purified by following the usual procedures. Reactivity ratios were determined by the method of Finemann and Ross ${ }^{12}$ and found to be $r_{1}=0.60$ for ST and $r_{2}=0.55$ for MMA. Harwood-Ritchey's run number ${ }^{13}$ was calculated from the reactivity ratios and the monomer feed composition data. Table I summarizes the results of characterization of the copolymer samples employed.

\section{Measurements}

The UV absorption measurements were carried out in spectrograde tetrahydrofuran and dioxane (Uvasol, E. Merck AG., Darmstadt), by a Zeiss DMR-21 UV Spectrometer. The temperature variation of UV absorption was measured in dioxane solution. The concentrations of ST monomer units in solution were about $5 \times 10^{-3}$ $\mathrm{mol} / l$. In the following the extinction coefficients are given in $l /(\mathrm{mol}-\mathrm{sty}$ rene unit) $\mathrm{cm}$.

\section{RESULTS AND DISCUSSION}

\section{UV Spectra of Polystyrenes and Their Dimer Model Compounds}

Figure 1 shows UV absorption spectra of isotactic and syndiotactic-rich polystyrenes and meso- and $d, l-2,4-d i p h e n y l p e n t a n e s$ in dioxane at various temperatures. There can be seen absorption peaks and shoulders at about $268\left(\lambda_{1}\right)$, $264\left(\lambda_{2}\right), 261\left(\lambda_{3}\right), 259\left(\lambda_{4}\right)$, and $254\left(\lambda_{5}\right) \mathrm{nm}$, as indicated by arrows in Figure 1A. The UV spectra of polymer samples show significant differences, depending on the microtacticity of the polymers. The maximum absorption peaks for I-PS and S-PS are different; absorbance of

Table II. Wavelength $\lambda$ and extinction coefficient $\varepsilon$ at peak and shoulder positions of polystyrenes and their dimer model compounds in dioxane solution at $25^{\circ} \mathrm{C}$

\begin{tabular}{|c|c|c|c|c|c|c|}
\hline \multirow{2}{*}{ Sample } & \multicolumn{3}{|c|}{$\lambda, \mathrm{nm}$} & \multicolumn{3}{|c|}{$\begin{array}{c}\varepsilon \times 10^{-2}, \\
l \mathrm{~mol}^{-1} \mathrm{~cm}^{-1}\end{array}$} \\
\hline & $\lambda_{1}$ & $\lambda_{3}$ & $\lambda_{4}$ & $\varepsilon_{1}$ & $\varepsilon_{3}$ & $\varepsilon_{4}$ \\
\hline S-PS & 268.5 & 261.5 & 259 & 1.557 & 2.084 & 2.053 \\
\hline I-PS & 268 & 261.5 & 259 & 1.463 & 2.006 & 2.021 \\
\hline$d, l$-isomer & 268 & 261 & 259 & 1.675 & 2.248 & 2.204 \\
\hline meso-isomer & 267.5 & 260.5 & 258.5 & 1.538 & 2.138 & 2.167 \\
\hline
\end{tabular}




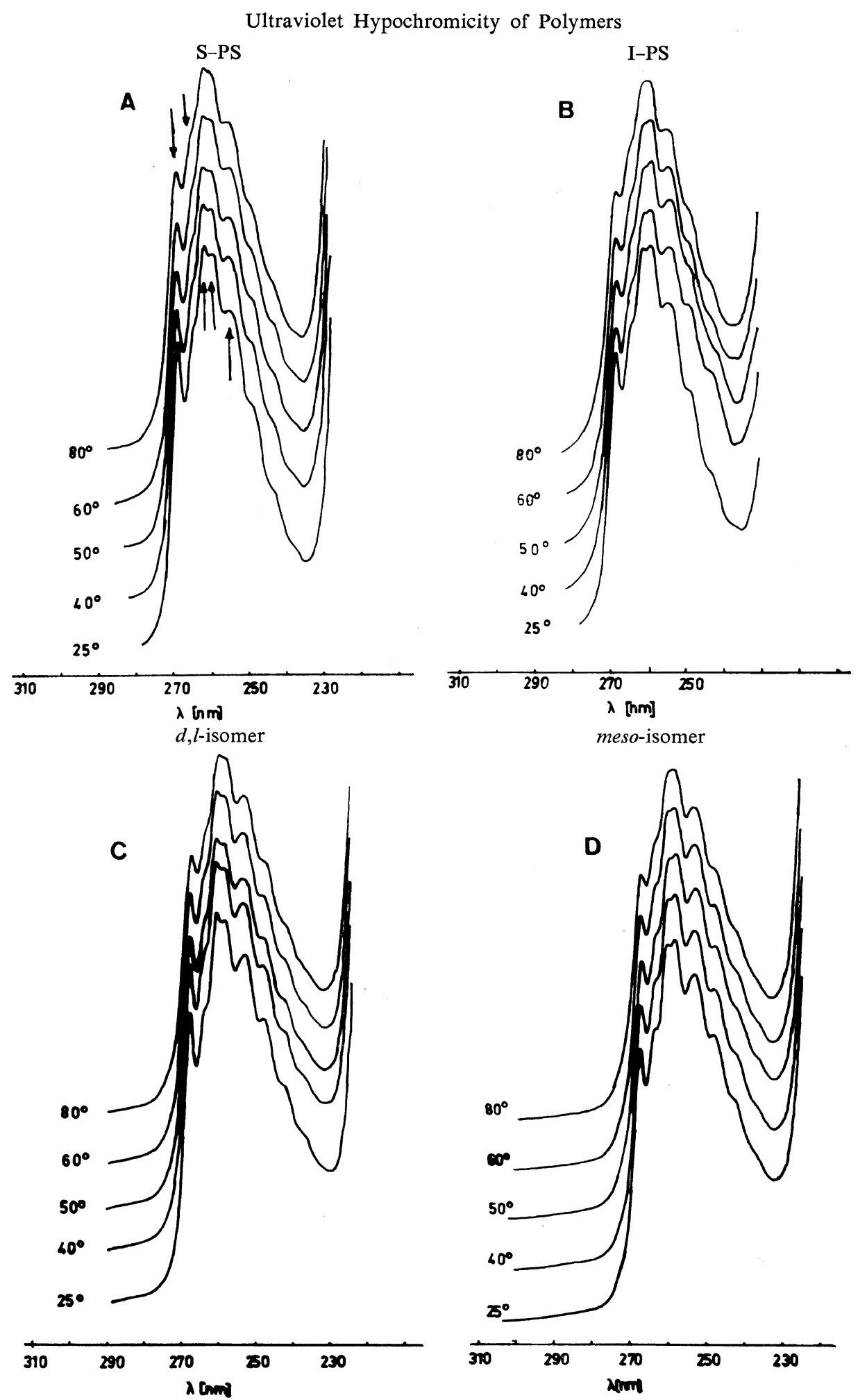

Figure 1. UV absorption spectra of isotactic (I-PS), syndiotactic-rich polystyrene (S-PS), meso-, and $d, 1-2,4-d i p h e n y l p e n t a n e s$ in dioxane at various temperatures. Figures indicate the measuring temperature.

Polymer J., Vol. 8, No. 3, 1976 
S-PS takes a maximum value at $261 \mathrm{~nm}$ and that of I-PS at $259 \mathrm{~nm}$. A similar deviation is observed also between the dimer model compounds. Table II lists the values of extinction coefficients at about 268,261, and $259 \mathrm{~nm}$ in dioxane solution at $25^{\circ} \mathrm{C}$. The extinction coefficients of the isotactic polymer and the mesoisomer are smaller than those of the syndiotacticrich polymer and the $d, l$-isomer, respectively. According to Vala and Rice, ${ }^{14}$ this is due to the so-called hypochromic effect caused by the anisotropic effect of phenyl rings. A similar hypochromic effect on the steric isomerism has been observed in the case of poly(methyl methacrylate) (PMMA). ${ }^{6 \mathrm{~b}, 6 \mathrm{c}}$ However, in the case of poly[(+)-2-methyl butyl methacrylate] the extinction coefficient of the isotactic polymer is larger than that of the syndiotactic one. ${ }^{6 c}$ The isotactic polymer chain is known to take a certain regular helical conformation even in solution. ${ }^{9}$ Such a helical conformation of the chain seems to play an important role in the UV absorption of the isotactic polymer, since the UV absorption is considered to reflect the local structure of the polymer chain. This explanation, however, is not sufficient in view of the fact that the same effect is observed for our dimer model compounds. Thus, the contribution of the helical conformation to UV absorption would be of minor one, if any. The mutual position in space of adjacent phenyl rings seems to controle the extinction coefficient as well as the shape and the form of the absorption spectrum.

\section{Temperature Dependence of Extinction Coefficient}

Figure 2 shows the temperature dependences of the extinction coefficients of the isotactic polymer and the meso-isomer. The coefficients decrease with increasing temperature. Each curve exhibits a small discontinuity between 40 and $50^{\circ} \mathrm{C}$. This phenomenon has been attributed to a certain conformational change of polystyrene in solution. ${ }^{5}$ For example, it has been speculated from light-scattering measurements that the destruction of helical turns in the polymer chain occurs above this temperature range. $^{5 \mathrm{a}}$ Meanwhile, the measurement of the half-width of the NMR peak of phenyl rings suggests that the temporarily stable structure of

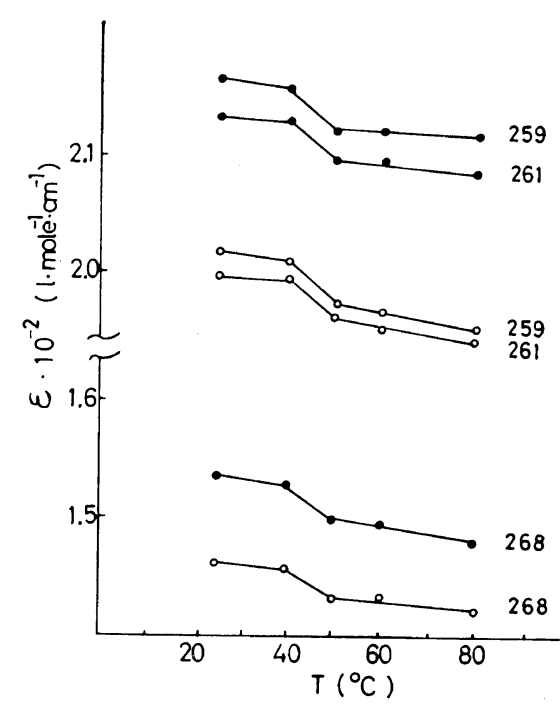

Figure 2. Temperature dependences of extinction coefficients of isotactic polystyrene $(\bigcirc)$ and meso2,4-diphenylpentane (O). Values described on each curve are wavelength $(\mathrm{nm})$.

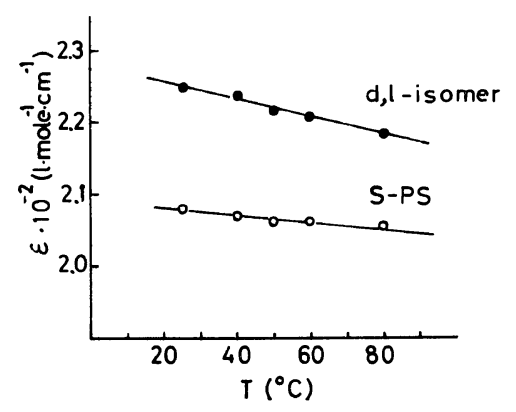

Figure 3. Temperature dependences of extinction coefficients of syndiotactic-rich polystyrene (O) and $d, l-2,4$-diphenylpentane $(\mathbf{)})$ at $261 \mathrm{~nm}$.

groups of chain units, which is created by the van der Waals forces between adjacent phenyl rings on polystyrenes, breakes up and reforms within this temperature range. ${ }^{5 c}$ This is related to the motions of the chain segments and can be attributed, in part, to the rotation of phenyl rings, Our results are in favor of the latter speculation, since the dimer model compounds is obviously unable to form a helical structure and yet it shows quantitatively the same temperature dependence of UV extinction coefficient as the isotactic polymer. On the other hand, the temperature dependences of $\varepsilon$ of the syn- 
diotactic-rich polymer and the $d, l$-isomer exhibit no detectable discontinuity as shown in Figure 3 (in the figure the value of $\varepsilon$ only at about $261 \mathrm{~nm}$ is plotted against temperature).

Effect of the Sequential Arrangement of Monomers

Preliminary UV absorption measurements were carried out to examine the dependence of extinction coefficient on the sequential arrangement of monomers. Three different model STMMA copolymers (SM-AL, SM-Block, and SM-50) with nearly equimolar composition and a mixture of radically prepared PMMA and SPS were used as reference samples. SM-Block and a mixture of PMMA and S-PS show the same UV spectra as S-PS, whereas the spectra of SM-AL and SM-50 are different from that of S-PS and even from each other; the extinction coefficients become smaller in the order of SM-Block, SM-50, and SM-AL. In addition, SM-Block takes the maximum absorbance at about $261 \mathrm{~nm}$, while SM-AL and SM-50 take at about $259 \mathrm{~nm}$ (see Table III). The difference in $\varepsilon$ values of SM-Block and SM-AL is as much as $0.4 \times 10^{2}\left(l \mathrm{~mol}^{-1} \mathrm{~cm}^{-1}\right)$ at about $261 \mathrm{~nm}$. This means that the largest hypochromic effect is found when the ST units are flanked by MMA units on both sides and it amounts to about $20 \%$ at $261 \mathrm{~nm}$.

Figure 4 shows UV spectra of SM-series copolymer samples with different ST contents. We can see the variation of the spectrum with ST content; when ST content is small it is similar to that for SM-AL and approaches that for SM-Block with increasing ST content. The values of extinction coefficients at the peak or shoulder positions are summarized in Table III. The extinction coefficients of random copolymer lie between those of block and alternating co-

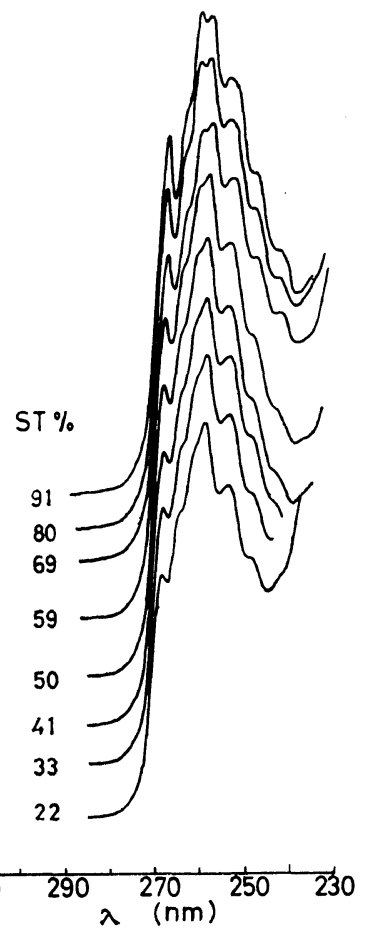

Figure 4. UV absorption spectra of random STMMA copolymers with different styrene contents in tetrahydrofuran at $25^{\circ} \mathrm{C}$. Values described on each spectrum are styrene content $(\mathrm{mol} \%)$.

Table III. Wavelength $\lambda$ and extinction coefficient $\varepsilon$ at peak and shoulder positions of ST-MMA copolymer samples in tetrahydrofuran solution at $25^{\circ} \mathrm{C}$

\begin{tabular}{|c|c|c|c|c|c|c|c|c|}
\hline \multirow{2}{*}{ Sample } & \multicolumn{4}{|c|}{$\lambda, \mathrm{nm}$} & \multicolumn{4}{|c|}{$\varepsilon \times 10^{-2}, l \mathrm{~mol}^{-1} \mathrm{~cm}^{-1}$} \\
\hline & $\lambda_{1}$ & $\lambda_{2}$ & $\lambda_{3}$ & $\lambda_{4}$ & $\varepsilon_{1}$ & $\varepsilon_{2}$ & $\varepsilon_{3}$ & $\varepsilon_{4}$ \\
\hline SM-20 & 269.3 & 265 & 262.5 & 259.5 & 1.102 & 1.383 & 1.660 & 1.796 \\
\hline SM-30 & 269.3 & 265 & 262.5 & 259.5 & 1.159 & 1.441 & 1.765 & 1.859 \\
\hline $\mathrm{SM}-40$ & 269.3 & 265 & 262.5 & 259.5 & 1.243 & 1.514 & 1.848 & 1.942 \\
\hline SM-50 & 269 & 265 & 262 & 259.5 & 1.326 & 1.566 & 1.921 & 1.979 \\
\hline SM-60 & 269 & 265 & 261.5 & 259.5 & 1.368 & 1.593 & 1.963 & 2.015 \\
\hline SM-70 & 269 & 265 & 261.5 & 259.5 & 1.410 & 1.613 & 1.974 & 2.036 \\
\hline SM-80 & 269 & 265 & 261.5 & 259.5 & 1.545 & 1.640 & 2.001 & 2.052 \\
\hline SM-90 & 269 & 265 & 261.5 & 259.5 & 1.587 & 1.665 & 2.071 & 2.022 \\
\hline S-PS & 269 & 265 & 261.5 & 259.5 & 1.598 & 1.675 & 2.086 & 2.030 \\
\hline SM-AL & 269.5 & 265.5 & 262 & 259 & 1.094 & 1.397 & 1.699 & 1.813 \\
\hline SM-Block & 269 & 265 & 261.5 & 259 & 1.585 & 1.667 & 2.073 & 2.024 \\
\hline
\end{tabular}


polymers. The values of the three extinction coefficients $\left(\varepsilon_{2}, \varepsilon_{3}\right.$, and $\left.\varepsilon_{4}\right)$ of sample SM-20 are smaller than the corresponding values of the alternating copolymer. This is ascribed to the experimental error, since the ST content in SM-20 is too small to measure the extinction coefficients accurately.

The measured extinction coefficients of random SM-series copolymers, $\varepsilon_{\mathrm{av}}$, can be approximately expressed in terms of the probabilities of the three possible triads each with a ST unit in the center: ${ }^{12 \mathrm{~b}}$

$$
\varepsilon_{\mathrm{av}}=f_{\mathrm{SSS}} \varepsilon_{\mathrm{SSS}}+f_{\mathrm{MSS}} \varepsilon_{\mathrm{MSS}}+f_{\mathrm{MSM}} \varepsilon_{\mathrm{MSM}}
$$

Here, $\mathbf{S}$ and $\mathbf{M}$ represent styrene and methyl methacrylate units, respectively. The probability of finding each triad in a random copolymer may be computed from the run number, which may also be deduced on the basis of copolymerization kinetics. ${ }^{13,15}$ Within the framework of the above approximation the extinction coefficient of homopolystyrene may be identified with $\varepsilon_{\mathrm{SSS}}$, and that of alternating copolymer with $\varepsilon_{\text {MSM }}$. Rearrangement of eq 1 yields

$$
\Delta \varepsilon=\varepsilon_{\mathrm{av}}-\left(f_{\mathrm{SSS}} \varepsilon_{\mathrm{SSS}}+f_{\mathrm{MSM}} \varepsilon_{\mathrm{MSM}}\right)=f_{\mathrm{MSS}} \varepsilon_{\mathrm{MSS}}
$$

Thus, $\Delta \varepsilon$ for a given wavelength should be proportional to the triad probability $f_{\text {MSs }}$ with the proportionality constant $\varepsilon_{\text {MSS }}$, if the triad approximation is valid. This proportionality is confirmed for the SM-series copolymers as revealed in Figure 5. The slopes give 1.440 and $2.030 \times 10^{2}\left[l \mathrm{~mol}^{-1} \mathrm{~cm}^{-1}\right]$ for $\varepsilon_{\mathrm{MSS}}$ at 269 and 259 $\mathrm{nm}$, respectively. This, in turn, means that the measured extinction coefficient of a given random ST-MMA copolymer allows us to evaluate the run number of the sample, if the three $\varepsilon$ values, $\varepsilon_{\mathrm{SSS}}, \varepsilon_{\mathrm{MSM}}$, and $\varepsilon_{\mathrm{MSS}}$ are known, as eq 1 shows.

Turning our attention to the problem in GPC and MCC fractionation, it is evident that the point-by-point composition of ST determined by UV detector will be always underestimated considerably, if the hypochromic effect is not taken correctly into account. In the case of copolymers with low compositional heterogeneity or in the case where the variation of point-by-point composition with elution volume is negligible, this effect may be corrected by knowing the percentage of hypochromicity of the whole

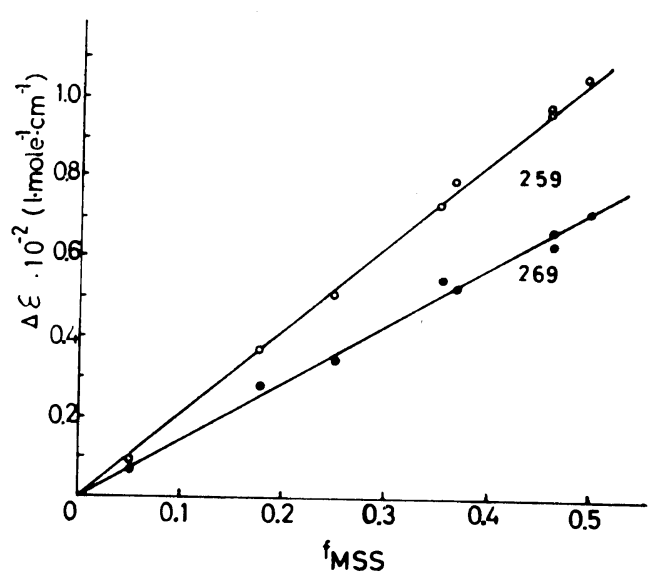

Figure 5. Plots of triad probability $f_{\text {MSS }}$ against $\Delta \varepsilon$ according to eq 2 for random ST-MMA copolymers (cf. Table III) at $269($ ) and $259 \mathrm{~nm}$ (O).

polymer sample. In the case where the variation of point-by-point composition with elution volume occurs, however, it is not easy to solve this problem precisely by some simple calculations, because it is impossible practically to know the run number at each elution volume. In practice, therefore, we would like to suggest that the UV calibration curve for determination of the point-by-point composition should not be made with homopolymer but with copolymer of a type similar to a smaple under fractionation. In fact, Probst, et al., ${ }^{16}$ have obtained good results for the fractionation of ST-butadiene radical copolymer samples containing 5 to $30 \%$ of ST.

Acknowledgements. We wish to thank the Deutsche Forschungsgemeinschaft and the Fonds der chemischen Industrie for financial support.

\section{REFERENCES}

1. H.-J. Cantow, J. Probst, and C. Stojanov, Kaut. Gummi, Kunstst., 21, 609 (1968).

2. J. R. Runyon, D. E. Barnes, J. F. Rudd, and L. H. Tung, J. Appl. Polym. Sci., 13, 2359 (1969).

3. K. F. O'Driscoll, W. Wertz, and A. Husar, J. Polym. Sci., Part A-1, 5, 2159 (1967).

4. R. J. Brüssau and D. J. Stein, Angew. Makromol. Chem., 12, 59 (1970).

5. (a) C. Reiss and H. Benoit, C.R. Acad. Sci. 
Paris, 253, 268 (1961); (b) G. Weill and C. Reiss, ibid., 257, 2816 (1963); (c) Kang-Jen Liu and R. Ullman, Polymer, 6, 100 (1965); (d) A. M. Liquori and F. Quadrifoglio, Polymer, 4, 448 (1963).

6. (a) M. D'Alagni, P. De Santis, A. M. Liquori, and M. Savino, J. Polym. Sci., Part B, 2, 925 (1964); (b) Kang-Jen Liu, J. S. Linowski, and R. Ullman, Makromol. Chem., 105, 18 (1967); (c) K. Ohara, Polymer J., 2, 109 (1971).

7. C. G. Overberger and P. V. Bonsignore, $J$. Amer. Chem. Soc., 80, 5427 (1958).

8. F. A. Bovey, F. P. Hood III, E. W. Anderson, and L. C. Snyder, J. Chem. Phys., 42, 3900 (1965).

9. M. Kobayashi, K. Akita, and H. Tadokoro, Makromol. Chem., 118, 324 (1968).
10. H. Ohnuma, T. Kotaka, and H. Inagaki, Polymer, 10, 501 (1969).

11. M. Hirooka, H. Yabuuchi, J. Iseki, and Y. Nakai, J. Polym. Sci., Part A-1, 6, 1381 (1968).

12. M. Finemann and S. D. Ross, J. Polym. Sci., 5, 269 (1950).

13. (a) H. J. Harwood and W. M. Ritchey, ibid., Part B, 2, 601 (1964); (b) H. J. Harwood, Angew. Chem., 77, 405 and 1124 (1965).

14. M. T. Vala and S. A. Rice, J. Chem. Phys., 39, 2348 (1963).

15. See, e.g., F. R. Mayo, Ber. Bunsenges. Phys. Chem., 70, 233 (1966).

16. J. Probst and H.-J. Cantow, Kaut. Gummi, Kunstst., 25, 11 (1972); Angew. Makromol. Chem., 35, 177 (1974). 